\title{
Integrability of strongly correlated electron systems on open chains
}

\author{
XIANG-Yu GE
}

In recent years, there has been a strong increase of interest in models of strongly correlated electron systems in condensed matter physics, in particular in integrable onedimensional models. Examples of such models are the one-dimensional Hubbard model, the supersymmetric $t-J$ model, the Hubbard-like model (Bariev's chain), the so-called extended Hubbard model (EKS model), the supersymmetric $U$ model and the eight-state supersymmetric $U$ model.

The study of integrable open-boundary conditions for strongly correlated electron models is very important for physical applications, especially for gaining insights into the behaviour of more realistic systems. The important ingredient in Sklyanin's boundary quantum inverse scattering method (QISM), which has played a key role in recent advances in the study of lattice models with open-boundaries, is a new algebraic structure, the reflection equation algebra.

In this thesis, a general graded reflection equation algebra is proposed and the corresponding graded (supersymmetry) boundary QISM is formulated. The formalism is applicable to all boundary lattice systems where an invertible $R$-matrix exists. As applications, some important integrable boundary conditions for various strongly correlated electron models with quantum superalgebra symmetries are constructed, including:

1. Presentation of integrable open-boundary conditions for the one-dimensional $q$ deformed supersymmetric Essler-Korepin-Schoutens (EKS) extended Hubbard model. Determination of three different diagonal boundary $K$-matrices and of nine classes of integrable open-boundary conditions. Construction of the exactly solvable Hamiltonian with open-boundary terms for quantum superalgebra $U_{q}(g l(2 \mid 2))$ symmetry;

2. Establishment of the quantum integrability of open-boundary conditions for the one-dimensional $q$-deformed supersymmetric $U$ model with quantum superalgebra $U_{q}(g l(2 \mid 1))$ symmetry. Determination of diagonal boundary $K$-matrices and of integrable open-boundary conditions. Solution of the boundary system by means of the coordinate space Bethe ansatz technique, and derivation of the Bethe ansatz equations;

Received 24th January, 2001

Thesis submitted to The University of Queensland, August 2000. Degree approved, November 2000. Supervisor: Professor Anthony J. Bracken.

Copyright Clearance Centre, Inc. Serial-fee code: 0004-9727/01 \$A2.00+0.00. 
3. Construction of nine classes of integrable open-boundary conditions for a twoparameter ( $q$-deformed) eight-state supersymmetric fermion model with quantum superalgebra $U_{q}(g l(3 \mid 1))$ symmetry. All classes are shown to be integrable. The boundary systems are solved by using the coordinate Bethe ansatz method, and Bethe ansatz equations given for all nine cases;

4. Consideration of the integrable open-boundary model of three-coupled one dimensional $X Y$ spin chains in the framework of the boundary QISM. Determination of diagonal boundary $K$-matrices and of a class of integrable open-boundary terms. The boundary model Hamiltonian is solved by using the coordinate Bethe ansatz method and Bethe ansatz equations derived.

Integrable boundary Kondo problems describing two impurities coupled to some one dimensional open chain are studied for specific couplings by using the graded boundary QISM. This is achieved by solving the graded reflection equations and finding the highly nontrivial non-c-number boundary $K$-matrices and the corresponding "singular" local monodromy matrices. Some important integrable models with boundary Kondo impurities are constructed, including:

5. Integrable open-boundary conditions for the $X X X$ model with impurities, studied by using the boundary QISM. The model Hamiltonian is diagonalized by means of the algebraic Bethe ansatz method and the Bethe ansatz equations are derived;

6. Integrable boundary Kondo impurities in two cases of the one-dimensional $q$ deformed $t-J$ models with $U_{q}(g l(2 \mid 1))$ and $U_{q}(s u(3))$ are studied by using the graded boundary QISM. The model Hamiltonians are solved by means of the algebraic Bethe ansatz method and the Bethe ansatz equations are obtained;

7. Integrable boundary Kondo impurities in the $q$-deformed EKS model with quantum superalgebra $U_{q}(g l(2 \mid 2))$ symmetry are studied by using the graded boundary QISM. The model Hamiltonian is diagonalized by means of the algebraic Bethe ansatz method and the Bethe ansatz equations are derived.

Department of Mathematics

The University of Queensland

Queensland 4072

Australia 